\title{
Hybrid Electrical/Mechanical Optimization Technique Using Time- Domain Modeling, Finite Element Method and Statistical Tools for Composite Smart Structures
}

\author{
Daniela Staiculescu ${ }^{1}$, Chisang You ${ }^{1}$, Lara Martin ${ }^{2}$, Woonbong Hwang ${ }^{3}$ and Manos Tentzeris ${ }^{1}$ \\ ${ }^{1}$ Georgia Institute of Technology, Atlanta, GA, 30332, U.S.A. \\ ${ }^{2}$ Motorola, Plantation, FL, 33322, U.S.A. \\ ${ }^{3}$ Pohang University of Science and Technology, Pohang, 790-784, Rep. of Korea
}

\begin{abstract}
The successful use of a Design of Experiments (DOE) and Response Surface Methods (RSM) approaches in a simultaneous electrical and mechanical optimization study for a load-bearing antenna structure is presented. The benchmarking geometry is a stacked patch antenna integrated in a sandwich structure made of composite laminates and Nomex honeycomb. The antenna is electromagnetically modeled in time domain and it is found that, for the chosen geometry, the honeycomb structure improves the gain of the antenna without affecting the bandwidth. The structure is then optimized using the same experiment that integrates both the electrical and mechanical (calculated with finite elements) parameters of the system. The simple factorial design is very simple to implement and gives a clear understanding of the system behavior, including the interaction between the mechanical changes and electrical performance thus allowing the engineer to integrate, for the first time, both the electrical and mechanical features of the system in the same optimization technique.
\end{abstract}

Index Terms - Time domain modeling, hybrid optimization, mechanical performance, composite smart structure.

\section{INTRODUCTION}

In the recent years, the need for improved structural efficiency and performance for 3D integrated structures, such as stacked antennas or micromachined cavities, has led to the embedding of the functions and components in load-bearing structural surfaces for aircraft applications [1-3]. The use of a composite roof structure in military vehicles with multiple antennas ensures high electrical performance in a light-weight and durable self-contained structure [4]. Through the innovative integration of antenna elements, amplifiers and ground plane, the reception quality and manufacturability of vehicle-mounted modules is expected to be significantly improved. The most important problem is that structurally effective materials cannot be used without reducing antenna efficiency. The present work aims to codesign electrically and structurally effective antenna structures, named composite smart structures (CSS). Design is focused electrically toward high gain and wide bandwidth, and mechanically toward high strength and stiffness. Stacked-patch microstrip antennas are commonly used due to their wideband performance, and sandwich structures composed of composites facesheets and
Nomex honeycomb are added to give good mechanical performance. The structure is first designed into a time domain full-wave (TLM-based) electromagnetic simulator and it is found that the addition of the honeycomb improves the gain of the antenna without affecting the bandwidth [5]. The mechanical performance is evaluated using a Finite Element Method (FEM) solver. Then, the electrical and mechanical analyses are incorporated into the Design of Experiments (DOE) and Response Surface Method (RSM) statistical optimization techniques, which give a thorough understanding of the system and, most importantly, give information such as how the electrical and mechanical performances are affected by each other, namely how they interact. Previous work [6] shows successful use of hybrid statistical techniques in microwave system analysis and optimization, but this is the first reported work on incorporating the mechanical performance into the same cosimulation. The system is optimized with respect to all these factors simultaneously, also giving the designer the flexibility to choose the goals and the weights of each of the optimized outputs.

\section{BenchMarking StRUCtURE}

The choice of the 3D benchmarking component is a stacked microstrip patch, chosen for its increased bandwidth due to the coupling of the two resonances for the two individual patches. The antenna layers and dimensions are shown in Fig. 1.

For enhanced mechanical performance, the antenna structure is sandwiched between two relatively dense and stiff facesheets bonded to either side of a low-density core [5]. The entire fabricated assembly is presented in Fig. 2. The Nomex honeycomb is made of Aramid Fiber Paper, formed in a honeycomb configuration and dipped in heat resistant resin. The cell size is $1 / 8$ ", and the density is $51 \mathrm{bs} / \mathrm{ft}^{3}$.

First, the antenna performance has been modeled in a timedomain TLM-based simulator. Preliminary simulations have demonstrated that the honeycomb structure and an effectively uniform medium with a 1.1 dielectric constant and zero loss tangent gives similar results for all the Nomex honeycomb product line. 


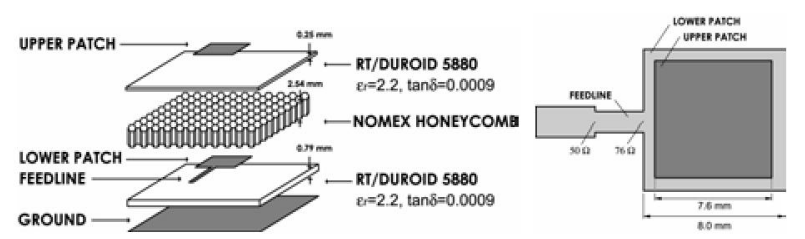

Fig. 1. Antenna layers and dimensions

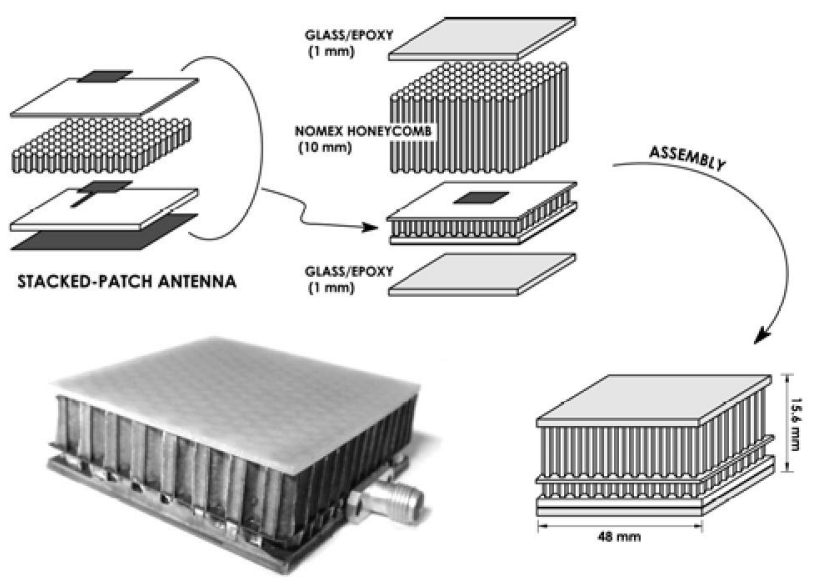

Fig. 2. Assembly procedure of CSS

The TLM simulation space spans $43 \times 59 \times 60$ cells using an adaptive grid. The maximum frequency used for the simulation is $15 \mathrm{GHz}$ and the excitation is a $1 \mathrm{~V}$ electric field source at the ports. The resonant frequency is $12.2 \mathrm{GHz}$. The performance was assessed and the simulator confirmed with microwave measurements of the test structure. The result of the comparison between measurements and simulations for the antenna insertion loss is shown in Fig. 3.

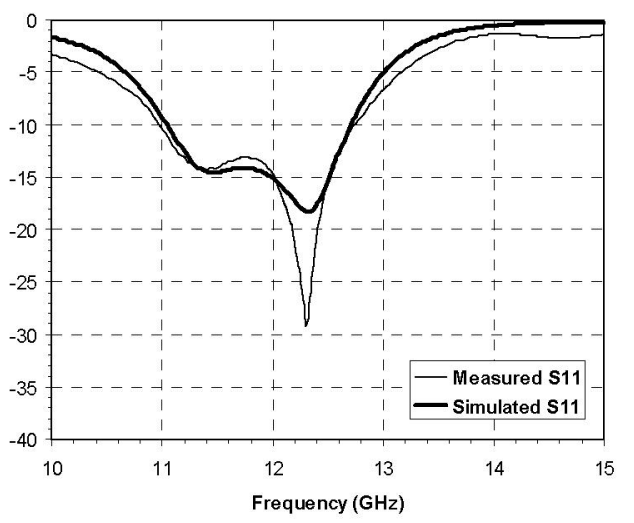

Fig. 3. Simulation vs. measurements for electrical performance

The mechanical analysis is performed in a commercial FEM simulator. The mechanical deflection is defined as the displacement, in millimeters, at the central load point when three-point bending is applied as shown in Fig. 4.

The two parameters under investigation for the hybrid optimization, which affect both the electrical and mechanical performance of the system, are the thicknesses of the Nomex honeycomb, $h$, and the thickness of the facesheet on the top of it, $t$. The design space for the two parameters has been chosen such that it represents physically realizable values and incorporates fabrication limitations and design rules. Also, since a very pronounced curvature is expected of the model, the range was chosen small enough to ensure the possibility to model the curvature with a second order model. The ranges for the two input variables are presented in TABLE I.

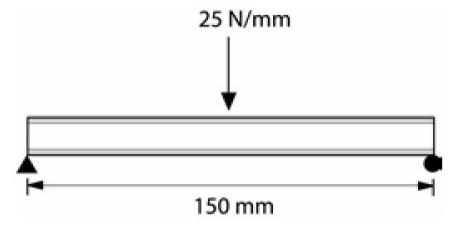

Fig. 4. Applied load for mechanical deflection evaluation

TABLE I

RANGES FOR THE INPUT VARIABLES

\begin{tabular}{|c|c|c|c|}
\hline Variable & Low value "-“ & High value "+" & Center point \\
\hline$t(\mathrm{~mm})$ & 1 & 1.5 & 1.25 \\
\hline$h(\mathrm{~mm})$ & 8.5 & 9.5 & 9 \\
\hline
\end{tabular}

The responses for the statistical models are the antenna gain at the resonant frequency $G$ and the mechanical deflection $D$.

The methodology used in the optimization of the antenna structure is presented as a flowchart in Fig. 5.

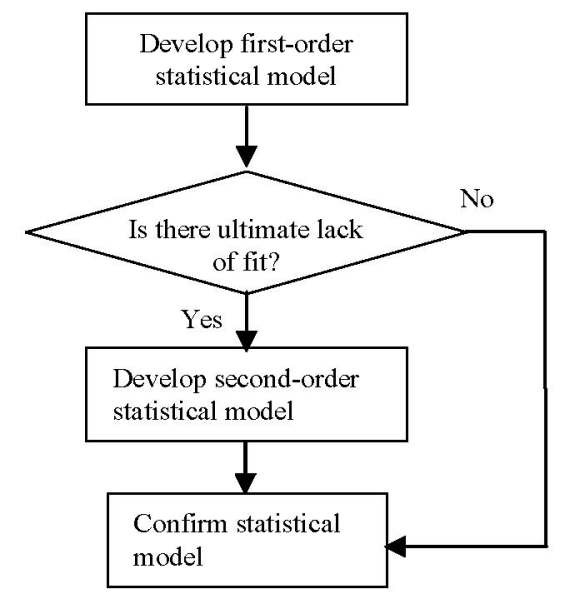

Fig. 5. Procedure for statistical model development

The experimentation method chosen for the first-order statistical model is a full factorial DOE with center points [7]. The factorial designs are used in experiments involving several factors where the goal is the study of the joint effects of the factors on a response. Prior knowledge of the analyzed system is required for choosing the factors and their studied ranges. The $2^{k}$ factorial design is the simplest one, with $k$ factors at 2 levels each. It provides the smallest number of runs for studying $k$ factors and is widely used in factor screening experiments [7]. In our case, the $2^{k}$ simulations had 
to be run for both electrical and mechanical analyses, but it is still a very small amount of simulations for obtaining generic design equations describing both the electrical and mechanical performance variation. Center points are defined at the center of the design space, as indicated in TABLE I, and increase the capability of investigating the validity of the model, including curvature in the response, and account for variation in the fabrication process of the structure. Since the statistical models are based on deterministic simulations, the variation of the center points were statistically simulated based on a $\pm 0.15 \mathrm{~mm}$ tolerance for $h, \mathrm{a} \pm 0.1 \mathrm{~mm}$ tolerance for $t$ and a $3 \sigma$ fabrication process. Specifically, center points were randomly generated assuming a mean equal to the exact center point value and a standard deviation equal to $0.03333 \mathrm{~mm}$ for $t$ and $0.05 \mathrm{~mm}$ for $h$.

Then, the model is checked for ultimate lack of fit, that is, if curvature might be present in the output response. If curvature in the response is detected, the analysis is extended to additional axial points indicated by the RSM method, which can account for curvature through second-order model development. Usually, these second-order models are reasonable approximations of the true functional relationship over relatively small regions. Once validated using statistical diagnostic tools, the models approximate the actual system within the defined design space.

In this case, a $2^{2}$ full factorial DOE was performed for the first-order statistical model and RSM was needed for the second-order statistical model. Once the models were validated for the model assumptions, the final statistical models were confirmed for prediction of the output variables and an optimization of the tested antenna performed.

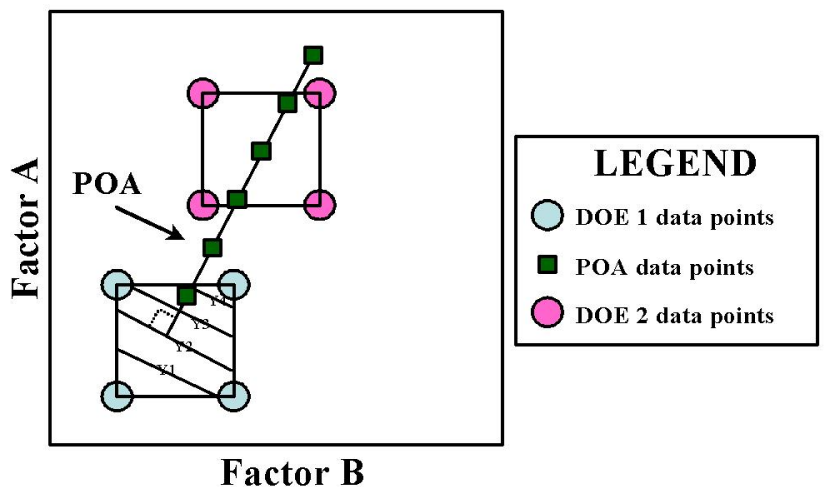

Fig. 6. Optimization using POA methodology for a simplified experimental design consisting of only two input variables.

A more sophisticated approach to optimize systems with large curvature is path of ascent (POA). In these cases, smaller intervals are chosen for the input variables in order to provide good models that account for the large curvature, and the optimal may be outside the initial design space. POA is applied to determine if there is a path for further optimization of the figures of merit outside the initial design space. Using this path, simulations are run until the optimum or a design rule limitation is reached. The optimum POA point is then used to identify another design space and then another full factorial DOE with center points is performed. The process is complete when the performance goal or optimum performance is achieved, as shown in Fig. 6. Results including POA for a more complex RF module will be presented at the conference.

\section{Statistical ANALYsis}

From the DOE, first-order statistical models were developed, which showed to have poor fit. Upon inspection of the statistical diagnostic tools used to validate assumptions of normality and equal variance, curvature was detected for both $G$ and $D$, with more extreme curvature for $G$. Even with the detected curvature for $D$, the model for $D$ was statistically significant at the $95 \%$ confidence level. Given the extreme curvature detected for $G$, the model for $G$ was not statistically verified as significant, testing just slightly lower than the $95 \%$ confidence level.

The following step was a second-order model development using RSM to attempt modeling the detected curvature in the figures of merit. The statistical models for $G$ and $D$ were both statistically significant at the $95 \%$ confidence level. Both $t$ and $\mathrm{h}$ and the interaction $\mathrm{t}^{*} \mathrm{~h}$ were included terms in the model for $G$, and both $\mathbf{t}$ and $\mathbf{h}$ were included terms in the model for $D$. Additionally the curvature was alleviated by including the second-order $h^{2}$ term for $G$ and by the second-order $t^{2}$ term for $D$. The model for $G$ was validated for the model assumption of normality and, for the model assumption of equal variance, only a slight decrease in variance with increasing $G$ was detected. Additionally, the model for $D$ was validated for both model assumptions of normality and equal variance. These models are given by (1)-(2).

$$
\begin{aligned}
& G=11.36+0.234\left(\frac{t-1.25}{0.25}\right)-0.258\left(\frac{h-9}{0.5}\right) \\
& -0.507\left(\frac{t-1.25}{0.25}\right)\left(\frac{h-9}{0.5}\right)-0.269\left(\frac{h-9}{0.5}\right)^{2} \\
& D=1.475-0.192\left(\frac{t-1.25}{0.25}\right)-0.061\left(\frac{h-9}{0.5}\right) \\
& +0.03\left(\frac{t-1.25}{0.25}\right)^{2}
\end{aligned}
$$

Before proceeding to the optimization, the models had to be confirmed. The confirmations of the models were performed for the following combination of parameters: $t=1.35 \mathrm{~mm}, h=$ $8.6 \mathrm{~mm}$. This configuration was simulated in the electromagnetic and mechanical simulators and was also predicted with the developed models. The results of the simulation, compared to the RSM 95\% confidence intervals defined by the lower and upper bounds for the predicted $\mathrm{G}$ and $\mathrm{D}$, are shown in TABLE II.

Because the simulation values fall into the $95 \%$ confidence intervals from the RSM, the RSM models were confirmed. with confirmation of the models, the RSM models given by (1)-(2) were accepted as the final models for optimization. 
TABLE II.

GAIN AND DEFLECTION FROM SIMULATION COMPARED TO THE RSM 95\% CONFIDENCE INTERVALS

\begin{tabular}{|c|c|c|}
\hline & $G$ & $D$ \\
\hline Simulation & 11.59 & 1.45 \\
\hline RSM lower bound & 11.15 & 1.42 \\
\hline RSM upper bound & 12.15 & 1.48 \\
\hline
\end{tabular}

\section{MODEL INTERPRETATION AND OPTIMIZATION}

Because the models are based on the identical model parameters, the models give the possibility to optimize the filter performance with respect to either figure of merit or both simultaneously allocating any weight factors to each one of them. They can also be used to predict the performance of the system for a specific configuration and to optimize using different weights for different applications and structures.

The final step in our study was the actual optimization of the benchmarking structure. The optimization goals chosen in this case were a resonant frequency maximum gain $G$ (weight $=0.6)$ and minimum deflection $D($ weight $=0.4)$. The surfaces for the two figures of merit as a function of the optimizing parameters are presented in Fig. 7. The optimization is done based on the plot in Fig. 8. The values that satisfied the two optimization conditions within the ranges presented in TABLE I were $t=1.5 \mu \mathrm{m}$ and $h=8.64 \mu \mathrm{m}$, leading to the optimized values of the two figures of merit of $G=12 \mathrm{~dB}$ and $D=1.36 \mathrm{~mm}$. The RSM optimized structure was simulated in the electromagnetic simulator and the values obtained for the output variables are $G=11.9 \mathrm{~dB}$ and $D=1.35 \mathrm{~mm}$. These simulation results agreed well with those predicted by the statistical models.

\section{CONCLUSIONS}

The DOE and RSM approaches have been combined with full-wave time-domain electrical and FEM mechanical simulations to perform for the first time the simultaneous optimization of the electrical and mechanical performance of a load-bearing antenna structure. The experiment was very simple to implement and provided a thorough understanding of the issues to be confronted to in the optimization process. The statistical analysis provided second order design equation including both electrical and mechanical figures of merit, then the optimization has been performed simultaneously by allocating arbitrary weight factors to each one of them. By extending this approach to carefully investigate the behavior of a complex system, such as a 3D multilayer module, the designer can save a lot of time, shorten the design cycle of added functions and achieve all aspects of the design process, both electrical, mechanical, even thermal in a simple and elegant manner and with a profound understanding of how all these aspects are affecting each other.
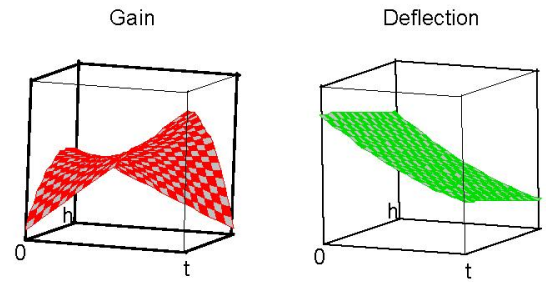

Fig. 7. Surfaces of possible solutions for optimized $G$ and $D$

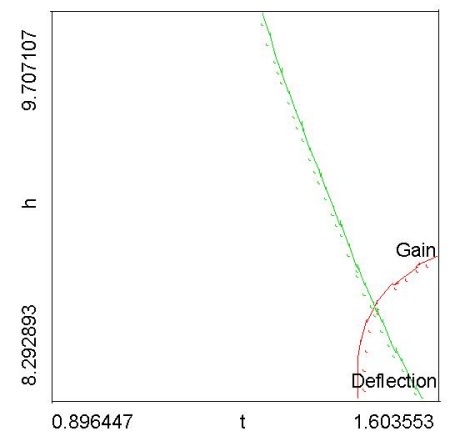

Fig. 8. Intersection of the surfaces represent the possible values of $t$ and $h$ that satisfy the optimization conditions.

\section{ACKNOWLEDGEMENT}

The authors wish to acknowledge the NSF CAREER ECS9984761, the NSF ECS-0313951, the Georgia Electronic Design Center (GEDC) and NSCS group at Postech for support with experimental results.

\section{REFERENCES}

[1] A. J. Lockyer, et al., "Design and Development of a Conformal Load-Bearing Smart-Skin Antenna: Overview of the AFRL Smart Skin Structures Technology Demonstration ( $\left.\mathrm{S}^{3} \mathrm{TD}\right)$ ", SPIE Smart Structures and Materials: Industrial and Commercial Application of Smart Structures Technologies, vol. 3674, pp. 410-424, 1999.

[2] A. J. Lockyer, et al., "A Qualitative Assessment of Smart Skins and Avionics/Structures Integration", SPIE Smart Structures and Materials: Smart Materials, vol. 2189, pp. 172-183, 1994.

[3] J. Tuss, et al., "Conformal Loadbearing Antenna Structure", 37th AIAA SDM Conference, pp. 836-843, 1996.

[4] Delphi Fuba Multiple Antenna Reception System, http://www.delphi.com.

[5] C.S. You, W. Hwang, "Design of Load-Bearing Antenna Structures by Embedding Technology of Microstrip Antenna in Composite Sandwich Structure", Composite Structures, Vol. 71, Issue 3-4, pp. 378-382, December 2005.

[6] N.Bushyager, D.Staiculescu, L.Martin, J.-H.Lee, N.Vasiloglou and M.M.Tentzeris, "Design and Optimization of 3D RF Modules, Microsystems and Packages Using Electromagnetic and Statistical Tools", Proc. of the 2004 IEEE-ECTC Symposium, pp.1412-1415, June 2004.

[7] J. Neter et al, "Applied Linear Statistical Models", $4^{\text {th }}$ Ed., The McGraw-Hill Companies, Chicago, 1996. 